\title{
Assessing biological aging: the origin of deficit accumulation
}

\author{
Arnold Mitnitski $\cdot$ Xiaowei Song • \\ Kenneth Rockwood
}

Received: 5 June 2013/Accepted: 8 July 2013/Published online: 17 July 2013

(C) The Author(s) 2013. This article is published with open access at Springerlink.com

\begin{abstract}
The health of individuals is highly heterogeneous, as is the rate at which they age. To account for such heterogeneity, we have suggested that an individual's health status can be represented by the number of health deficits (broadly defined by biological and clinical characteristics) that they accumulate. This allows health to be expressed in a single number: the frailty index (FI) is the ratio of the deficits present in a person to the total number of deficits considered (e.g. in a given database or experimental procedure). Changes in the FI characterize the rate of individual aging. The behavior of the FI is highly characteristic: it shows an age specific, nonlinear increase, (similar to Gompertz law), higher values in females, strong associations with adverse outcomes (e.g., mortality),
\end{abstract}

Electronic supplementary material The online version of this article (doi:10.1007/s10522-013-9446-3) contains supplementary material, which is available to authorized users.

A. Mitnitski $(\bowtie) \cdot X$. Song $\cdot$ K. Rockwood

Department of Medicine, Dalhousie University, Suite 229-5790 University Ave., Halifax, NS B3H 1V7, Canada e-mail: arnold.mitnitski@dal.ca

A. Mitnitski

Department of Mathematics and Statistics, Dalhousie University, Halifax, NS, Canada

K. Rockwood

Division of Geriatric Medicine, QEII Health Science Centre, Suite 1421-5955 Veterans' Memorial Lane,

Halifax, NS B3H 2E1, Canada and a universal limit to its increase (at FI 0.7). These features have been demonstrated in dozens of studies. Even so, little is known about the origin of deficit accumulation. Here, we apply a stochastic dynamics framework to illustrate that the average number of deficits present in an individual is the product of the average intensity of the environmental stresses and the average recovery time. The age-associated increase in recovery time results in the accumulation of deficits. This not only explains why the number of deficits can be used to estimate individual differences in aging rates, but also suggests that targeting the recovery rate (e.g. by preventive or therapeutic interventions) will decrease the number of deficits that individuals accumulate and thereby benefit life expectancy.

Keywords Health status - Deficit accumulation · Frailty $\cdot$ Fitness-frailty continuum $\cdot$ Mortality · Stochastic process

\section{Introduction}

Aging is a complex phenomenon but even so shows remarkable regularities in such characteristics as survival probability and mortality rates. Although these regularities can be observed reliably at the population level (the most famous being the Gompertz law of mortality), they are masked at the individual level by inter-personal variability. Individuals age in a variety of ways, making attractive the idea that they differ in their biological age. 
The estimation of biological age is difficult: how to integrate biological markers into such a measure of biological age is not clear. Recent attempts have been based on a battery of biological (performance) markers, used to construct an index of physical fitness age from 13 performance measures (Kimura et al. 2012). Many commentators find it attractive to assess biological aging using physiological measures that change with age; even so, the prospects for success with this approach are controversial (Ingram et al. 2001; Kulminski et al. 2009). There is some evidence however that a wide range of health related variables, and not just laboratory measures, can be used to assess biological age if they are combined appropriately. Such variables are readily available in large clinical and epidemiological databases (Mitnitski et al. 2005; Goggins et al. 2005; Yashin et al. 2007a, 2013; Gu et al. 2009; Theou et al. 2013). One such tool, the frailty index (FI), has been used to incorporate a range of health deficits (including biological ones) that people accumulate during their life course (Mitnitski et al. 2001; Kulminski et al. 2007; Yashin et al. 2007a).

While different individuals face a variety of different health problems, there is a common denominatorthe number of health problems increases with aging and increases faster in those individuals whose health is poor (Yashin et al. 2007a; Kulminski et al. 2007). Ageing develops gradually and starts from small changes in health (Kirkwood 2005) which accumulate across the adult life course (Rockwood et al. 2011). While many of the variables, when considered in isolation from each other, have only small effects on health, their cumulative effect becomes significant (Mitnitski et al. 2001, 2002; Kulminski et al. 2007). These cumulative effects can be quantified by combining health related variables (either biological or clinical, including even those self-reported) in a socalled fitness/frailty index or more often just a FI. This term reflects its extensive use in medicine, where the increased vulnerability of older adults to adverse outcomes is referred to as frailty (Fulop et al. 2010; Rockwood and Mitnitski 2011; Clegg et al. 2013).

Such measures have been investigated in both epidemiological surveys and clinical databases (Jones et al. 2004; Mitnitski et al. 2005; Woo et al. 2006; Rockwood et al. 2007, 2011; Kulminski et al. 2006; Dupre et al. 2009; Yang and Lee 2010; Singh et al. 2012; Eeles et al. 2012). These indices have been shown to be useful indicators of ageing, good predictors of adverse outcomes such as worsening health (Mitnitski et al. 2006; Fallah et al. 2011), poor response to vaccination (Ridda et al. 2009), institutionalization (Jones et al. 2005; Rockwood et al. 2007) and death (Jones et al. 2005; Mitnitski et al. 2005; Kulminski et al. 2007; Yashin et al. 2007a; Eeles et al. 2012; Singh et al. 2012). Recently this approach has been extended to animal models (Parks et al. 2012).

Of some interest, the properties of the FIs depend more on the number of deficits from which the FIs are comprised rather than on their nature (Rockwood et al. 2006; Rockwood and Mitnitski 2011) reflecting that health related variables are rarely independent. Given this interdependence, the rate of increase in the accumulation of deficits has been proposed as an estimate of the rate of aging (Mitnitski et al. 2001). That has some support: in general, the FI characterises individual health across the fitness-frailty continuum from the fittest (those who compared to others at their age, have accumulated just a few health problems) to the frailest people who, having accumulated many more problems than have others of their age, are the most vulnerable to stresses. In consequence, the fit are less prone, and the frail are more prone to adverse outcomes such as major illnesses, disabilities and mortality. The behaviour of the FI is highly characteristic: it accumulates exponentially with age (resembling Gompertz law), is strongly associated with mortality and has a distribution that changes with age but still has a limit of about 0.7 , beyond which further deficit accumulation is incompatible with life (Rockwood and Mitnitski 2006; Bennett et al. 2013).

The individual age trajectories of the FI show stochastic dynamic behaviour (Mitnitski et al. 2006, 2012; Fallah et al. 2011). Importantly, even though, on average, deficits accumulate over time, individual trajectories can either increase (indicating worsening of heath) or decrease. This indicates that health improvement is not just possible, but quite common, at least for a short run, likely reflecting medical interventions, changes in life styles, etc.

Despite the advances that have been made in understanding aging systems, the complexity of aging challenges researchers, especially those who are rooted in the success of the reductionist approach. Progress in considering multiple aging processes requires a systems biology approach (Jazwinski 2002; Tacutu et al. 2010a; Kirkwood 2011; Yashin et al. 2012). This is based on mathematical modeling, which makes it 
possible to apply mathematical insights and apparatus developed in other scientific fields, as commonly is done in other scientific areas, including physics, engineering, operations research etc.

Here, we consider a general stochastic model of how deficit accumulation results from interactions between two processes: the process of (broadly construed) environmental stresses, which cause damage in multiple systems of the organism, and that of damage control and recovery. Both processes are intrinsically stochastic, as there are always a multiplicity of factors that cannot be controlled. The model we employ here is based on a queuing theory approach (Erlang 1917; Gross and Harris 1998) and reflects a formal analogy between the number of deficits that the individuals have accumulated and the length of a queue in the queuing system such as a server. The model allows an understanding of the major patterns in deficits accumulation observed in many studies by our group (e.g., Mitnitski et al. 2001, 2005; Rockwood and Mitnitski 2006; Song et al. 2010) and others (GarcíaGonzález et al. 2009; Yang and Lee 2010). In particular, we show that the age-associated decline in the recovery rate (or the associated increase in the average time of recovery) together with the stochastic nature of environmental challenges explain the major patterns of deficits accumulation which all groups have observed across the life course.

\section{Model}

Outline of the model

Let consider the process of environmental challenges imposed by stresses on the organism as a stochastic Poisson process, with intensity rate $\lambda$. The average time interval between the consecutive stresses is thus $1 / \lambda$. In reality, such challenges are of many different natures, arising from individual exposures to perturbations in the climate, solar activity, weather, pollution, stressful social events, disease outbreaks, etc. The interval between the challenges $(1 / \lambda)$ can span from seconds to months; most cannot be measured. Let $W$ be the average time of recovery from such environmental damage in people of the same chronological age. Generally, the time of recovery is also variable, but has a minimum scale of days, spanning up to several months, depending on the nature of the specific damage and the organism's ability to recover. The ability of the organism to recover depends on the individual's health or the so-called reserve capacity, itself related to their genetic profile (Christensen et al. 2006; Yashin et al. 2013). Clearly too, an individual's health is related to the state of the health care in the society, so that not all environmental influence is malign. Despite all this, the time of recovery is age dependent, presumably reflecting subclinical (even microscopic) tissue, cellular and subcellular damage (Howlett and Rockwood 2013).

Queuing theory and Little's Law

The schema described above is structurally equivalent to what is well known in stochastic queuing theory-a mathematical discipline that aims to explain how the length of a queue is related to intensity of the steam of arrivals to the system, and to the systems' priority schedule, and service and waiting times. Queuing theory is widely employed across multiple applications in communications, computer architecture, operation management, to name just a few. The queuing system is usually modeled by a system of differential equations (the Kolmogorov equations). Their structure depends on specific assumptions of the model (e.g., single server or a network of servers; stationary or non-stationary arrivals, different priory schedules, etc.). Despite the great complexity of queuing equations, there is a general and simple relationship between the essential characteristics of queuing systems, known as Little's Law, which recently celebrated its 50th anniversary (Little 1961, 2011). This law states that the average number of items in a queuing system $(L)$ equals the average arrival rate $(\lambda)$ multiplied by the average waiting time of an item in the system, $W$.

$L=\lambda W$

Reformulating Little's Law in terms of our setting, we suggest that the average number of deficits present in an individual $(L)$ equals the rate of environmental stresses $\lambda$, times the average recovery time $W$.

Deficits accumulate, indicating that recovery time increases with age

The importance of this relationship is that it states that the average recovery time is proportional to the 
average number of deficits that the individual possesses. Here, the coefficient of proportionality $(\lambda)$ is a global characteristic of the environment. In general, Little's Law makes is possible to calculate one of the parameters in Eq. (1) by knowing two others. In some cases, however, even knowing one parameter may help to estimate the second one under the conditions that the third parameter does not change. Here, it suggests that even if during the life course the intensity of the environmental stresses remained constant, the average recovery time would still increase with age. This offers a potentially key insight, i.e. that the kinetics of the deficit accumulation with age is the same as the kinetics of recovery time. The kinetics of deficit accumulation is known to be of an exponential type (Mitnitski et al. 2005; Rockwood et al. 2011) with the exponent parameter typically close to 0.03 . This is illustrated in Fig. 1, which shows age-specific (crosssectional) average trajectories for the 9 waves of the National Population Health Survey of Canadian aged $20+$ years, over 16 years of follow-up, repeated every 2 years. Note that the overlaid trajectories virtually coincide and can be fitted with the exponential function with slope $0.035( \pm 0.02)$ (the solid line). There is about a threefold increase in the number of deficits during three decades after age 60 , and about an order of magnitude increase from age 20 to 100 years. Given the exponential increase in the number of deficits with age, by virtue of Little's Law we can say that the average recovery time increases exponentially with age, and with the same parameter $k=0.035$. In other words, the average $W$ satisfies the following differential equation:

$d W / d t=k W$

where $k=0.035$ is the constant slope estimated empirically from the data.

Changes in the distribution of the number of deficits

Because Little's Law operates only with the averages, the changes in the distribution of the number of deficits between age groups cannot be seen just from it alone. The patterns of the distribution, however, could be understood from the following simple considerations, consistent with queuing theory. When the recovery time is low (i.e. when the rate of recovery is high), the damage caused by the environmental stresses is

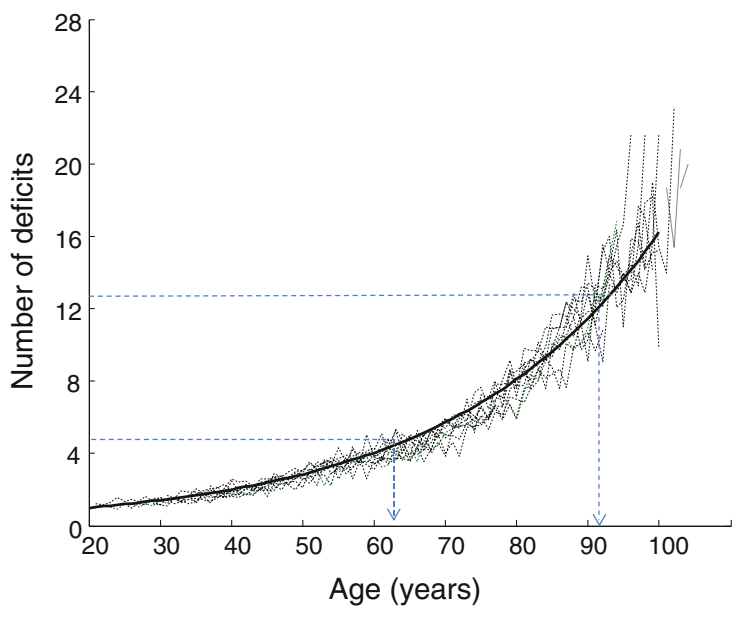

Fig. 1 Age trajectories of the mean number of deficits. Thin lines are the cross-sectional data for the nine consecutive twoyears cycles plotted against age. The solid line is the best exponential fit with the exponent of $0.035( \pm 0.02)$

quickly recovered, without persisting deficits. This is the case for young people, most of whom have no or a few deficits. This situation is similar to queuing systems with only "light traffic" (or "light utilization"), which show highly asymmetrical density distributions for the length of queue. Indeed, in younger people, the distribution of the number of deficits is highly asymmetrical (Fig. 2 stars and the dashed line for the individuals between 20 and 35 years old). In contrast, when the recovery rate slows, so that recovery time is long, deficits accumulate with age. Likewise, the number of people with zero deficits diminishes. Figure 2 also shows the density distribution of the number of deficits in an older group of individuals aged 75-100 years. The fitting function for the older individuals (the solid line) is well represented by the gamma density function, as is described in "heavy traffic" queuing models (Kyprianou 1972).

\section{Discussion}

In this paper, we have proposed a general and simple stochastic model to explain how the number of deficits present in individuals can be represented by the product of the intensity of environmental stresses to the average recovery time (in accordance with Little's Law). The exponential increase in the number of 


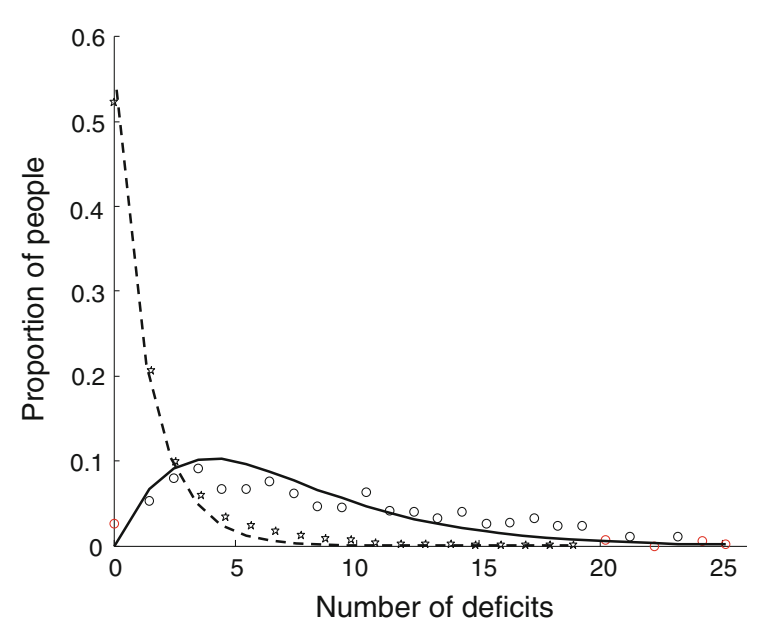

Fig. 2 Distribution of the number of deficits for the two age groups: 20-30 years old (stars are the observational data and the dashed line is the exponential fit with the exponent -0.6) and 75-100 years old (circles are the observational data and the solid line is the gamma fit with the shape and scale parameters of 2.1 and 3.2 , respectively)

health deficits with age directly corresponds to the exponential increase of recovery time, as does the changes with age in the distributions of the deficits. Fast recovery largely prevents deficits from being accumulated, so that the distribution remains highly asymmetrical (most people have no or few deficits). With a decline in the recovery rate, and a corresponding increase of recovery time, fewer people have no deficits and the mode of the distribution shifts to the right. For these reasons, recovery time is the fundamental parameter, which characterizes the health of individuals. Likewise, its population averages even characterize the health of the nations (Theou et al. 2013) as we discuss below.

A large body of evidence can relate an increase in recovery time to age-associated damages and diseases (Franceschi et al. 2000; Rattan 2003; Gurtner et al. 2008; Akushevich et al. 2013; Yanai et al. 2011). The mechanisms by which recovery occurs are complex and involve a number of processes, at different levels of the organism, from the DNA repair response (Moskalev et al. 2012), to repair of chromosomal damage (Nicholls et al. 2011) to autophagy (Couve and Schmachtenberg 2011; Fortini and Dogliotti 2010; Vicencio et al. 2008), degradation of repair capacity (Koga et al. 2011), and a host of others (Tacutu et al. 2010b; Howlett and Rockwood 2013; Yashin et al. 2013). This multiplicity of specific mechanisms responsible for age-related decline in the recovery rate likely corresponds to decline in flexibility (Fabre et al. 2007) or loss of stress resistance with aging seen with decline in allostatic adaptation (Yashin et al. 2007b, c, 2013). The decline in so many specific mechanisms has been characterized as "shrinkage of the homeodynamic space", due to "the stochastic occurrence and progressive accumulation of molecular damage" (Rattan 2012, 2013).

This age associated increase in recovery time can be regarded as a manifestation of the decline of vitality as was postulated by Strehler and Mildvan (1960), although in their model vitality declines linearly whereas the rate of recovery declines exponentially. Decline in the rate of recovery is also in line with the general systems biological mechanism of critical slowing down. Indicators of critical slowing down have been postulated as a means of broadly ranking complex systems, from resilient to fragile (Scheffer 2010; Veraart et al. 2011). We propose that the FI can be considered as such an indicator, in that it indeed provides a means of ranking individuals across grades of fitness and frailty (Rockwood and Mitnitski 2007).

Our model leaves aside the question why the time of recovery increases with age. Being hesitant to answer this question now, we can simply speculate that it reflects an increasing metabolic cost of maintenance (Kirkwood and Holliday 1979; Kirkwood 2011). In general, the entropy of the environment is higher than the entropy of the organism; the latter is far from equilibrium (Nicolis and Prigogine 1979). In consequence, keeping such non-equilibrium conditions is possible for only a limited time (limited by the life span). The incorporation of such a mechanism in the model is a matter for future investigation.

Our results should be interpreted with caution. First, this is a simplistic model, although this is commonly the price paid to make any model tractable. In this regard, there are many ways to make the model more realistic, for example by considering nonPoisson and even non-stationary environments, to allow damage events not be independent of each other. Third, recovery time includes three features that are distinguishable: avoidance of damage by a successful mechanism (which in any case is indistinguishable from instantaneous recovery); resistance (e.g. in a stationary environment, a given exposure such as blunt force trauma might result in bruising in a younger person, but fracture in an older one, which then will 
require a longer recovery period) and recovery itself. Relatedly, even though Little's Law holds independently of the priority schedule of dealing with damage, the distributions of the number of deficits will differ for different priority protocols. For example, under heavy traffic conditions (i.e., advanced age) if the strategy of the organism is to deal first with the most important problems, rather than in a way entirely determined by their order of their appearance (i.e. their "arrival", in queuing terms) this will lead to distributions of deficit number with heavy tails (i.e. more people will have more things wrong). Systems with such priority scheduling recently have been investigated in human dynamics (Barabási 2005; Vázquez et al. 2006) to explain emergence of heavy-tailed distribution in the queues. Also, deficits can emerge in clusters, e.g., more than one deficit might emerge at the same time. This can be addressed under the "multiserver" version of the model, which allows simultaneous acquisition of stresses. The Kolmogorov equations will be different from those for a single server system. Even so, the averages will still follow Little's Law, which holds independently of the structure of the system. Finally, in contrast to some advanced mathematical models of how health changes during aging (Yashin et al. 2007b, c, 2012, 2013) our model does not include mortality- here we concentrated exclusively on the model being conditional on survival. On the other hand, the number of deficits has proven to be a strong predictor of mortality, and always superior in this regard to chronological age (Mitnitski et al. 2005; Kulminski et al. 2007). For these reasons, we believe that considering these processes separately seems a good approximation of a more general process that includes longitudinal changes in deficits accumulation and mortality.

Despite these limitations, our model makes clear why and how health deficits accumulate with age, providing a systemic mechanism underlying the origin of biological ageing. The model captures the stochastic nature of the environmental stresses that cause damage (Gavrilov and Gavrilova 2006) and shows how an increase in recovery time explains the major patterns of deficit accumulation with aging we so far observed in all datasets. The model can be fine-tuned as new data on biological markers or performance measures become more widely available. In this way, it can organize a framework for better understanding now hidden mechanisms of how health deteriorates during aging.

Our model explains not only the major patterns in deficit accumulation in individuals but also clarifies differences in deficit accumulation between countries with different socioeconomic conditions. The average time of recovery at the level of population depends on the health care system and the latter is related to health care expenditures, as recently demonstrated by Theou et al. (2013). That study reported on more than 36,000 community dwelling people aged 50 and older from 15 European countries. In higher income countries, people had about 3 fewer deficits than in lower-income countries; similarly, the deficit number was negatively correlated with both gross domestic product $(r=$ $-0.79)$ and health expenditure $(r=-0.63)$ (Theou et al. 2013). A finding of a similar wealth-health gradient was reported by Semyonov et al. (2013).

The number of deficits individuals accumulate can be modifiable either by medical interventions, changes in the lifestyle, diet and exercise- all these factors are associated with the life span extension because the number of deficits diminishes in the individual who make such changes (Woo et al. 2006; Hubbard et al. 2009a, b; Fallah et al. 2009) and as it becomes clear, because they reduce the time of recovery.

\section{Conclusions}

The age associated increase in recovery time results in the accumulation of deficits as people grow older. This framework not only explains why the number of deficits can be used to estimate individual differences in aging rates but also suggests that targeting the recovery rate should decrease the number of deficits that individuals accumulate, and thereby improve life expectancy.

Acknowledgments This project was sponsored by an operating Grant from the Canadian Institutes of Health Research, no. MOP24388. The National Population Health Study was carried out by Statistics Canada. The authors obtained access to the data through an agreement with the Atlantic Research Data Centre of Statistics Canada, which obliged them to operate, for these purposes only, as "deemed employees" of Statistics Canada. Statistics Canada officials reviewed the analyses to ensure that confidentiality had not been breached and that the data were weighted properly to produce uniform estimates across reports. In no other way did any sponsor have influence over the data analyses. 
Conflict of interest $\mathrm{AM}$ and $\mathrm{KR}$ have applied to various Canadian government schemes that support commercialization of university research related to the FI.

Open Access This article is distributed under the terms of the Creative Commons Attribution License which permits any use, distribution, and reproduction in any medium, provided the original author(s) and the source are credited.

\section{References}

Akushevich I, Kravchenko J, Ukraintseva S, Arbeev K, Yashin AI (2013) Recovery and survival from aging-associated diseases. Exp Gerontol 48:824-830

Barabási AL (2005) The origin of bursts and heavy tails in human dynamics. Nature 435(7039):207-211

Bennett S, Song X, Mitnitski A, Rockwood K (2013) A limit to frailty in very old, community-dwelling people: a secondary analysis of the Chinese longitudinal health and longevity study. Age Ageing 42:372-377

Christensen K, Johnson TE, Vaupel JW (2006) The quest for genetic determinants of human longevity: challenges and insights. Nat Rev Genet 7:436-448

Clegg A, Young J, Iliffe S, Rikkert MO, Rockwood K (2013) Frailty in elderly people. Lancet 381(9868):752-762

Couve E, Schmachtenberg O (2011) Autophagic activity and aging in human odontoblasts. J Dent Res 90:523-528

Dupre ME, Gu D, Warner DF, Yi Z (2009) Frailty and type of death among older adults in China: prospective cohort study. BMJ 338:b1175. doi:10.1136/bmj.b1175

Eeles EM, White SV, O'Mahony SM, Bayer AJ, Hubbard RE (2012) The impact of frailty and delirium on mortality in older inpatients. Age Ageing 41:412-416

Erlang AK (1917) Solution of Some Problems in the Theory of Probabilities of Significance in Automatic Telephone Exchanges. Elektrotkeknikeren 13

Fabre JM, Wood RH, Cherry KE, Su LJ, Cress ME, King CM, deVeer MJ, Ellis R, Jazwinski SM (2007) Age-related deterioration in flexibility is associated with health-related quality of life in nonagenarians. Louisiana Healthy Aging Study. J Geriatr Phys Ther 30:16-22

Fallah N, Mitnitski A, Middleton L, Rockwood K (2009) Modeling the impact of sex on how exercise is associated with cognitive changes and death in older Canadians. Neuroepidemiology 33:47-54

Fallah N, Mitnitski A, Searle S, Gill TM, Gahbauer E, Rockwood K (2011) Transitions in frailty status in older adults in relation to mobility: a multi-state modeling approach employing a deficit count. J Am Geriatr Soci 59:524-529

Fortini P, Dogliotti E (2010) Mechanisms of dealing with DNA damage in terminally differentiated cells. Mutat Res 685:38-44

Franceschi C, Valensin S, Bonafè M, Paolisso G, Yashin AI, Monti D, De Benedictis G (2000) The network and the remodeling theories of aging: historical background and new perspectives. Exp Gerontol 35:879-896

Fulop T, Labri A, Witkowski JM, McElhaney J, Loeb M, Mitnitski A, Pawelec G (2010) Aging, frailty and age-related diseases. Biogerontology 11:547-563
García-González JJ, García-Peña C, Franco-Marina F, Gutiérrez-Robledo LM (2009) A frailty index to predict the mortality risk in a population of senior Mexican adults. BMC 9:47

Gavrilov LA, Gavrilova NS (2006) Reliability theory of aging and longevity. In: Masoro EJ, Austad SN (eds) Handbook of the biology of aging, 6th edn. Academic Press, Amsterdam, pp 3-42

Goggins WB, Woo J, Sham A, Ho SC (2005) Frailty index as a measure of biological age in a Chinese population. J Gerontol A Biol Sci Med Sci 60:1046-1051

Gross D, Harris CM (1998) Fundamentals of queuing theory. Wiley, New York

Gu D, Dupre ME, Sautter J, Zhu H, Liu Y, Yi Z (2009) Frailty and mortality among Chinese at advanced ages. J Gerontol B Psychol Sci Soc Sci 64:279-289

Gurtner GC, Werner S, Barrandon Y, Longaker MT (2008) Wound repair and regeneration. Nature 453:314-321

Howlett SE, Rockwood K (2013) New horizons in frailty: ageing and the deficit scaling problem. Age Ageing 42:416-423

Hubbard RE, Fallah N, Searle SD, Mitnitski A, Rockwood K (2009a) Impact of exercise in community-dwelling older adults. PLoS ONE 4:e6174

Hubbard RE, Searle SD, Mitnitski A, Rockwood K (2009b) Effect of smoking on the accumulation of deficits, frailty and survival in older adults: a secondary analysis from the Canadian Study of Health and Aging. J Nutr Health Aging 13:468-472

Ingram DK, Nakamura E, Smucny D, Roth GS, Lane MA (2001) Strategy for identifying biomarkers of aging in long-lived species. Exp Gerontol 36:1025-1034

Jazwinski SM (2002) Biological aging research today: potential, peeves, and problems. Exp Gerontol 37:1141-1146

Jones DM, Song X, Rockwood K (2004) Operationalizing a frailty index from a standardized comprehensive geriatric assessment. J Am Geriatr Soc 52:1929-1933

Jones D, Song X, Mitnitski A, Rockwood K (2005) Evaluation of a frailty index based on a comprehensive geriatric assessment in a population based study of elderly Canadians. Aging Clin Exp Res 17:465-471

Kimura M, Mizuta C, Yamada Y, Okayama Y, Nakamura E (2012) Constructing an index of physical fitness age for Japanese elderly based on 7-year longitudinal data: sex differences in estimated physical fitness age. Age (Dordr) 34:203-214

Kirkwood TB (2005) Understanding the odd science of aging. Cell 120:437-447

Kirkwood TB (2011) Systems biology of ageing and longevity. Philos Trans R Soc Lond B Biol Sci 366:64-70

Kirkwood TB, Holliday R (1979) The evolution of ageing and longevity. Proc R Soc Lond B Biol Sci 205:531-546

Koga H, Kaushik S, Cuervo AM (2011) Protein homeostasis and aging: the importance of exquisite quality control. Ageing Res Rev 10:205-215

Kulminski A, Yashin A, Arbeev K, Akushevich I, Ukraintseva S, Land K, Manton K (2006) Accumulation of heath disorders as a systemic measure of aging: findings from the NLTCS data. Mech Ageing Dev 125:840-848

Kulminski A, Ukraintseva SV, Akushevich I, Arbeev KG, Land K, Yashin AI (2007) Accelerated accumulation of health 
deficits as a characteristic of aging. Exp Gerontol 42:963-970

Kulminski AM, Arbeev KG, Culminskaya IV, Ukraintseva SV, Christensen K, Yashin AI (2009) Health-related phenotypes and longevity in Danish twins. J Gerontol A Biol Sci Med Sci 64:1-8

Kyprianou EK (1972) The quasi-stationary distributions of queues in heavy traffic. J Appl Prob 9:821-831

Little JDC (1961) A proof for the queuing formula: $\mathrm{L}=\lambda \mathrm{W}$. Operation Research 9:383-387

Little JDC (2011) Little's law as viewed on its 50th anniversary. Operation Research 59:536-549

Mitnitski AB, Mogilner AJ, Rockwood K (2001) Accumulation of deficits as a proxy measure of aging. Sci World J $1: 323-336$

Mitnitski AB, Graham JE, Mogilner AJ, Rockwood K (2002) Frailty, fitness and late-life mortality in relation to chronological and biological age. BMC Geriatr 2:1

Mitnitski A, Song X, Skoog I, Broe GA, Cox JL, Grunfeld E, Rockwood K (2005) Relative fitness and frailty of elderly men and women in developed countries and their relationship with mortality. J Am Geriatr Soc 53:2184-2189

Mitnitski A, Bao L, Rockwood K (2006) Going from bad to worse: a stochastic model of transitions in deficit accumulation, in relation to mortality. Mech Ageing Dev 127:490-493

Mitnitski A, Song X, Rockwood K (2012) Trajectories of changes over twelve years in the health status of Canadians from late middle age. Exp Gerontol 47:893-899

Moskalev AA, Shaposhnikov MV, Plyusnina EN, Zhavoronkov A, Budovsky A, Yanai H, Fraifeld VE (2012) The role of DNA damage and repair in aging through the prism of Koch-like criteria. Ageing Res Rev 12:661-684

Nicholls C, Li H, Wang JQ, Liu JP (2011) Molecular regulation of telomerase activity in aging. Protein Cell 2:726-738

Nicolis G, Prigogine I (1979) Irreversible processes at nonequilibrium steady states and Lyapounov functions. Proc Natl Acad Sci USA 6:6060-6061

Parks RJ, Fares E, Macdonald JK, Ernst MC, Sinal CJ, Rockwood K, Howlett SE (2012) A procedure for creating a frailty index based on deficit accumulation in aging mice. J Gerontol A Biol Sci Med Sci 67:217-227

Rattan SIS (2003) Continuous remodeling as a key to aging and survival: an interview with Claudio Franceschi. Biogerontology 4:329-334

Rattan SI (2012) Biogerontology: from here to where? The Lord Cohen Medal Lecture-2011. Biogerontology 13:83-91

Rattan SIS (2013) Healthy ageing, but what is health? Biogerontology 14 (in press)

Ridda I, Macintyre CR, Lindley R, Gao Z, Sullivan JS, Yuan FF, McIntyre PB (2009) Immunological responses to pneumococcal vaccine in frail older people. Vaccine 27: $1628-1636$

Rockwood K, Mitnitski A (2006) Limits to deficit accumulation in elderly people. Mech Ageing Dev 127:494-496

Rockwood K, Mitnitski A (2007) Frailty in relation to the accumulation of deficits. J Gerontol A Biol Sci Med Sci 62: 722-727

Rockwood K, Mitnitski A (2011) Frailty defined by deficit accumulation and geriatric medicine defined by frailty. Clin Geriatr Med 27:17-26
Rockwood K, Mitnitski A, Song X, Steen B, Skoog I (2006) Long-term risks of death and institutionalization of elderly people in relation to deficit accumulation at age 70. J Am Geriatr Soc 54:975-979

Rockwood K, Abeysundera MJ, Mitnitski A (2007) How should we grade frailty in nursing home patients? J Am Med Dir Assoc 8:595-603

Rockwood K, Mitnitski A, Song X (2011) Changes in relative fitness and frailty across the adult lifespan: evidence from the Canadian National Population Health Survey. CMAJ 59:814-821

Scheffer M (2010) Complex systems: foreseeing tipping points. Nature 467:411-412

Semyonov M, Lewin-Epstein N, Maskileyson D (2013) Where wealth matters more for health: the wealth-health gradient in 16 countries. Soc Sci Med 81:10-17

Singh I, Gallacher J, Davis K, Johansen A, Eeles E, Hubbard RE (2012) Predictors of adverse outcomes on an acute geriatric rehabilitation ward. Age Ageing 41:242-246

Song X, Mitnitski A, Rockwood K (2010) Prevalence and 10 -year outcomes of frailty in older adults in relation to deficit accumulation. J Am Geriatr Soc 58:681-687

Strehler BL, Mildvan AS (1960) General theory of mortality and aging. Science 132:14-21

Tacutu R, Budovsky A, Wolfson M, Fraifeld VE (2010a) MicroRNA-regulated protein-protein interaction networks: how could they help in searching for pro-longevity targets? Rejuvenation Res 13:373-377

Tacutu R, Budovsky A, Fraifeld VE (2010b) The NetAge database: a compendium of networks for longevity, agerelated diseases and associated processes. Biogerontology 11:513-522

Theou O, Brothers TD, Rockwood MR, Haardt D, Mitnitski A, Rockwood K (2013) Exploring the relationship between national economic indicators and relative fitness and frailty in middle-aged and older Europeans. Age Ageing

Vázquez A, Oliveira JG, Dezsö Z, Goh KI, Kondor I, Barabási AL (2006) Modeling bursts and heavy tails in human dynamics. Phys Rev E: Stat, Nonlin, Soft Matter Phys 73(3 Pt 2):036127

Veraart AJ, Faassen EJ, Dakos V, van Nes EH, Lürling M, Scheffer M (2011) Recovery rates reflect distance to a tipping point in a living system. Nature 481:357-359

Vicencio JM, Galluzzi L, Tajeddine N, Ortiz C, Criollo A, Tasdemir E, Morselli E, Ben Younes A, Maiuri MC, Lavandero S, Kroemer G (2008) Senescence, apoptosis or autophagy? When a damaged cell must decide its path-a mini-review. Gerontology 54:92-99

Woo J, Goggins W, Sham A, Ho SC (2006) Public health significance of the frailty index. Disabil Rehabil 28:515-521

Yanai H, Budovsky A, Tacutu R, Fraifeld VE (2011) Is rate of skin wound healing associated with aging or longevity phenotype? Biogerontology 12:591-597

Yang Y, Lee LC (2010) Dynamics and heterogeneity in the process of human frailty and aging: evidence from the U.S. older adult population. J Gerontol B Psychol Sci Soc Sci 65B:246-255

Yashin AI, Arbeev KG, Kulminski A, Akushevich I, Akushevich L, Ukraintseva SV (2007a) Cumulative index of elderly disorders and its dynamic contribution to mortality and longevity. Rejuvenation Res 10:75-86 
Yashin AI, Arbeev KG, Kulminski A, Akushevich I, Akushevich L, Ukraintseva SV (2007b) Health decline, aging and mortality: how are they related? Biogerontology 8:291-302

Yashin AI, Arbeev KG, Akushevich I, Kulminski A, Akushevich L, Ukraintseva SV (2007c) Stochastic model for analysis of longitudinal data on aging and mortality. Math Biosci 208:538-551

Yashin AI, Arbeev KG, Akushevich I, Kulminski A, Ukraintseva SV, Stallard E, Land KC (2012) The quadratic hazard model for analyzing longitudinal data on aging, health, and the life span. Phys Life Rev 9:177-188

Yashin AI, Arbeev KG, Wu D, Arbeeva LS, Kulminski A, Akushevich I, Culminskaya I, Stallard E, Ukraintseva SV (2013) How lifespan associated genes modulate aging changes: lessons from analysis of longitudinal data. Front Genet 4:3 